\title{
Do the Electrons and Ions in X\#Ray Clusters Share the Same Temperature?
}

\section{Citation}

Fox, David C., and Abraham Loeb. 1997. "Do the Electrons and lons in X\#Ray Clusters Share the Same Temperature?" The Astrophysical Journal 491 (2): 459-66. https://doi.org/10.1086/305007.

\section{Permanent link}

http://nrs.harvard.edu/urn-3:HUL.InstRepos:41393413

\section{Terms of Use}

This article was downloaded from Harvard University's DASH repository, and is made available under the terms and conditions applicable to Other Posted Material, as set forth at http:// nrs.harvard.edu/urn-3:HUL.InstRepos:dash.current.terms-of-use\#LAA

\section{Share Your Story}

The Harvard community has made this article openly available.

Please share how this access benefits you. Submit a story.

\section{Accessibility}


The Astrophysical JouRnal, 491:459-466, 1997 December 20

(C) 1997. The American Astronomical Society. All rights reserved. Printed in U.S.A.

\title{
DO THE ELECTRONS AND IONS IN X-RAY CLUSTERS SHARE THE SAME TEMPERATURE?
}

\author{
David C. Fox ${ }^{1}$ AND Abraham LoEb \\ Astronomy Department, Harvard University, 60 Garden Street, Cambridge, MA 02138 \\ Received 1997 June 26; accepted 1997 July 31
}

\begin{abstract}
The virialization shock around an X-ray cluster primarily heats the ions, since they carry most of the kinetic energy of the infalling gas. Subsequently, the ions share their thermal energy with the electrons through Coulomb collisions. We quantify the expected temperature difference between the electrons and ions as a function of radius and time, based on a spherical self-similar model for the accretion of gas by a cluster in an $\Omega=1, h_{50}=1$ universe. Clusters with X-ray temperatures $T_{\mathrm{X}}=4-10 \times 10^{7} \mathrm{~K}$ show noticeable differences between their electron and ion temperatures at radii $\gtrsim 2 \mathrm{Mpc}$. High-resolution spectroscopy with future X-ray satellites such as Astro-E may be able to determine the ion temperature in intracluster gas from the width of its X-ray emission lines and compare it to the electron temperature as inferred from the free-free emission spectrum. Any difference between these temperatures can be used to date the period of time that has passed since the infalling gas joined the cluster.
\end{abstract}

Subject headings: galaxies: clusters: general — intergalactic medium $-\mathrm{X}$-rays: galaxies

\section{INTRODUCTION}

The standard technique for measuring the total mass in clusters of galaxies from X-ray observations assumes that the cluster gas is in hydrostatic and thermal equilibrium (see, e.g., Jones \& Forman 1992). Hydrodynamic simulations often maintain the assumption of thermal equilibrium and show that while the gas is typically at hydrostatic equilibrium in the cores of clusters, its state is perturbed in their outer parts (Navarro, Frenk, \& White 1995; Evrard, Metzler, \& Navarro 1996). In this paper we address the question of whether the gas in these outer regions should actually be in thermal equilibrium as assumed. To see why the cluster gas might deviate from thermal equilibrium, we should first examine how the gas obtains its high X-ray temperature.

As fresh material joins the stationary cluster gas, it converts its kinetic energy of infall into heat through a shock. Since most of the inertia of the infalling gas is carried by the ions, they acquire most of the dissipated energy and heat to about twice the virial temperature of the cluster. The electrons, on the other hand, remain cold after the shock, and their temperature slowly rises as a result of Coulomb collisions with the hot ions. Thermal equilibrium will only be achieved if the temperature equilibration time between the ions and the electrons is shorter than the age of the cluster. In fact, the ratio of the equilibration time to the age of the universe is of order unity, $\sim\left(\Omega_{b} h_{50} / 0.06\right)\left(T / 10^{8} \mathrm{~K}\right)^{3 / 2}(\delta /$ $178)^{-1}$, for the characteristic overdensity $\delta$ and temperature $T$ in cluster shocks, where $\Omega_{b}$ is the baryonic density parameter and $h_{50}$ is the Hubble constant in units of $50 \mathrm{~km} \mathrm{~s}^{-1}$ $\mathrm{Mpc}^{-1}$. This implies that the electron and ion fluids might not achieve complete thermal equilibrium within the cluster lifetime.

A difference between the electron and ion temperatures was invoked as a potential explanation for the increase in the inferred baryon fraction in the outer parts of A2163

\footnotetext{
${ }^{1}$ Also Physics Department, Harvard University.
}

(Markevitch et al. 1996). The $A S C A$ data show a sharp drop in the electron temperature beyond $2 h_{50}^{-1} \mathrm{Mpc}$ in this cluster. The gas distribution, determined by ROSAT, is well described by a $\beta$-model. Assuming hydrostatic equilibrium, a dark matter distribution with the same profile as the gas was strongly ruled out by the temperature measurements, and models in which the dark matter fell off much more rapidly were only marginally allowed. If, however, the electron temperature is lower than the ion temperature, the actual gas pressure is higher than the value inferred under the assumption of thermal equilibrium. Markevitch et al. (1996) point out that this possibility might be real, since the equilibration timescale is comparable to the typical time since the last merger.

In principle, the deviation from thermal equilibrium could result either from smooth accretion or from mergers of clumps. For simplicity, we focus on smooth accretion in this paper. Making use of the versatility of a semianalytic approach, we employ a self-similar model of spherical accretion to examine the regime of validity of the assumption of thermal equilibrium between the electrons and ions. This approach is similar to that of Itoh (1978), who considered thermal equilibration in supernova remnants using a selfsimilar blast-wave model. We assume that Coulomb collisions are the only coupling mechanism between the temperatures of electrons and ions. It can be shown that a homogeneous plasma with isotropic, monotonically decreasing distribution functions is stable against plasma instabilities (Rosenbluth 1965). Additional coupling might be provided by plasma instabilities in more complicated conditions, but we ignore them in this discussion.

In $\S 2$ we describe the method of the calculation. In $\S 3$ we relate the parameters of our model to the conditions in typical clusters. In $\S 4$ we describe the details of the numerical calculations. In $\S 5$ we quantify the effects of the approximations made in our model. Finally, $\S 6$ discusses the results and the possibility of detecting the difference between the ion and electron temperatures with future $\mathrm{X}$-ray telescopes. Throughout the paper we assume a 
density parameter $\Omega=1$, a Hubble constant $h_{50}=1$, and a hydrogen mass fraction $X=76 \%$.

\section{METHOD OF CALCULATION}

\subsection{Collision Timescales}

We first consider collisional relaxation processes in plasmas consisting of several species of particles. Spitzer (1962) showed that each species achieves a Maxwellian distribution on a timescale of the order of the collision time,

$$
\begin{aligned}
t_{x x} & =\frac{m_{x}^{1 / 2}(3 k T)^{3 / 2}}{5.71 \pi n e^{4} Z^{4} \ln \Lambda} \\
& =3.60 \times 10^{8} \mathrm{yr} \frac{\left(T / 10^{8} \mathrm{~K}\right)^{3 / 2}}{\left(n / 10^{-3} \mathrm{~cm}^{-3}\right)} \frac{A^{1 / 2}}{Z^{4} \ln \Lambda},
\end{aligned}
$$

where $A=m_{x} / m_{p}$ is the particle mass of the species $x$ in units of the proton mass, $Z e$ is the particle charge, and $\ln \Lambda$ is the Coulomb logarithm, given by

$$
\ln \Lambda \approx\left\{\begin{array}{c}
37.8+\ln \left(\frac{T}{10^{8} \mathrm{~K}}\right)-\frac{1}{2} \ln \left(\frac{n}{10^{-3} \mathrm{~cm}^{-3}}\right) \\
\text { for electrons } \\
40.5+\frac{3}{2} \ln \left(\frac{T}{10^{8} \mathrm{~K}}\right)-\frac{1}{2} \ln \left(\frac{n}{10^{-3} \mathrm{~cm}^{-3}}\right) \\
\text { for ions }
\end{array}\right.
$$

Thus, the collision time for protons, $t_{p p} \approx\left(m_{p} / m_{e}\right)^{1 / 2} t_{e e}$, is longer by a factor of 43 than that for electrons, $t_{e e}$.

Spitzer also found that the exchange of energy in collisions between test particles and field particles, with Maxwellian distributions of temperatures $T$ and $T_{f}$, is governed by the equation

$$
\frac{d T}{d t}=\frac{T_{f}-T}{t_{\mathrm{eq}}},
$$

where the equilibration timescale $t_{\mathrm{eq}}$ depends on the related particle masses $m$ and $m_{f}$ and charge numbers $Z$ and $Z_{f}$ as well as the density of field particles $n_{f}$ and is given by

$$
\begin{aligned}
t_{\mathrm{eq}}= & \frac{3 m m_{f}}{8(2 \pi)^{1 / 2} n_{f} Z^{2} Z_{f}^{2} e^{4} \ln \Lambda}\left(\frac{k T}{m}+\frac{k T_{f}}{m_{f}}\right)^{3 / 2} \\
= & 1.86 \times 10^{8} \mathrm{yr} \frac{A_{f}}{Z^{2} Z_{f}^{2} A^{1 / 2} \ln \Lambda} \\
& \times \frac{\left(T / 10^{8} \mathrm{~K}\right)^{3 / 2}}{\left(n_{f} / 10^{-3} \mathrm{~cm}^{-3}\right)}\left(1+\frac{T_{f}}{T} \frac{A}{A_{f}}\right)^{3 / 2} .
\end{aligned}
$$

For electron-ion collisions, the Coulomb logarithm for electrons is appropriate. For collisions between protons and ions of similar mass, such as $\mathrm{He}^{++}$, the equilibration timescale is comparable to $t_{p p}$. For electrons and ions, the equilibration timescale is greater than $t_{p p}$ by a factor of order $\left(m_{p} / m_{e}\right)^{1 / 2}$.

Thus, equilibrium in an electron-proton plasma is achieved in several stages. First, the electrons reach a Maxwellian distribution with temperature $T_{e}$ on a timescale $t_{e e}$. Then, on a longer timescale, $t_{p p} \sim\left(m_{p} / m_{e}\right)^{1 / 2} t_{e e}$, the protons reach a Maxwellian distribution with temperature $T_{p}$. Finally, the two temperatures equalize on a timescale of order $t_{e p} \sim\left(m_{p} / m_{e}\right) t_{e e}$. We would like to consider a fully ionized plasma of hydrogen and helium, which is only slightly more complicated. All three ion timescales, $t_{\mathrm{He}^{++}}$, $t_{p p}$, and $t_{\mathrm{He}^{++} \mathrm{He}^{++}}$, are much shorter than the electron-ion equilibration timescale. Therefore, when considering electron-ion equilibration, we can regard the ions as sharing a single temperature $T_{i}$. In this case,

$$
\frac{d T_{e}}{d t}=\frac{T_{i}-T_{e}}{t_{e i}},
$$

where the energy exchange rate between the ions and the electrons is just the sum of the rates owing to exchange with the two ion species,

$$
1 / t_{e i}=1 / t_{e p}+1 / t_{e \mathrm{He}^{++}} .
$$

We will generally be in the regime in which $T_{e} / T_{i} \gg$ $m_{e} / m_{p}$, where the contribution from each species to $t_{e i}^{-1}$ is proportional to $n_{f} Z_{f}^{2} / A_{f}$. For both $p$ and $\mathrm{He}^{++}$, this factor simply equals the field particle density $n_{f}$. Thus, in the case of a fully ionized plasma of hydrogen and helium,

$$
t_{e i}=7.97 \times 10^{9} \mathrm{yr} \frac{\left(T_{e} / 10^{8} \mathrm{~K}\right)^{3 / 2}}{\left(n_{i} / 10^{-3} \mathrm{~cm}^{-3}\right) \ln \Lambda},
$$

where $n_{i}$ is the total ion number density. This timescale differs from $t_{e p}$ only by a factor of $n_{p} / n_{i}$.

\subsection{Self-similar Model for Spherical Accretion}

For simplicity, we consider spherical accretion of gas onto an X-ray cluster in an $\Omega=1$ universe. Bertschinger (1985) found that an initial top-hat mass perturbation results in a self-similar solution, for both purely collisional and purely collisionless fluids, as well as for a single collisional fluid in a potential well dominated by a collisionless fluid (i.e., $\Omega_{b} \ll \Omega=1$ ).

We would like to adapt this last model to the case of a fully ionized plasma of hydrogen and helium, under the gravitational influence of collisionless dark matter. The gas in Bertschinger's model is considered adiabatic, and we retain this simplifying assumption. Thus, we neglect radiative cooling, a well-justified assumption for the outer parts of clusters where the free-free cooling time is much longer than the Hubble time. We also assume that there exists a magnetic field that is sufficiently strong to suppress thermal conduction, yet small enough to have a negligible dynamical effect. These conditions are indeed satisfied by the inferred magnetic field strength $\sim 0.1-1 \mu \mathrm{G}$ in cluster environments (Kim et al. 1990; Kim, Tribble, \& Kronberg 1991; Rephaeli 1988). The general case of a stationary shock in a fully ionized plasma with conduction is complicated by the fact that the electron thermal speed exceeds the shock speed, allowing the electrons to preheat the plasma ahead of the shock (Shafranov 1957; Zeldovich \& Raizer 1967). If conduction is suppressed by a magnetic field, however, we will see that electron-ion equilibrium can be treated entirely locally, which simplifies the problem considerably.

The self-similar model is divided into three regions: preshock, shock, and postshock. In the preshock region, the gas is taken to be cold, so that pressure is neglected. In our model, we take the plasma to be fully ionized even before the shock, so it cannot literally have zero temperature (e.g., photoionization by the UV background would heat the gas to $\sim 10^{4}-10^{5} \mathrm{~K}$ ). However, as long as the temperature is sufficiently low that the initial pressure is dynamically insig- 
nificant compared to gravity (i.e., $T \ll 10^{7} \mathrm{~K}$ for $\mathrm{X}$-ray clusters), this will not affect the preshock behavior. In this case, the acceleration is due to gravity alone, and thus the preshock boundary conditions for the gas density, pressure, and velocity $(\rho, p$, and $v)$ are the same as in the case of a single, cold, neutral gas.

The behavior of the gas at the shock is determined by the shock jump conditions for $\rho, p$, and $v$. These conditions derive from the conservation of total mass, momentum, and energy. Since we neglect conduction, the equations relating the preshock density, pressure, and velocity, $\rho_{1}, p_{1}$, and $v_{1}$, to the postshock values, $\rho_{2}, p_{2}$, and $v_{2}$, are the same for an ionized plasma as for a single monatomic gas. Since the preshock flow is cold, $p_{1}$ is negligible compared to $p_{2}$, and the shock is strong.

Even though the relation between preshock and postshock conditions is unchanged, the self-similar solution could still be altered by the finite shock thickness. The thickness of collisional shocks is typically of the order of the mean free path, which, for Coulomb collisions, is proportional to $T^{2}$. For the ions, whose postshock temperature is high, the mean free path in the postshock region may be large. In the hottest clusters, at $z=0$, it can become a nonnegligible fraction of the shock radius, $r_{s}$, which characterizes the scale of the solution. This could break the self-similarity of the solution. In the presence of the magnetic field mentioned above, however, a collisionless shock, which can be much thinner than a mean free path, is more likely. We will neglect the finite thickness of the shock in our model.

The shock jump conditions determine the mean temperature, $\bar{T}$, given the mass per particle, $\mu m_{p}$. However, in order to find $T_{i}$ and $T_{e}$ separately, we must consider the shock region in more detail. Inside the shock, collisions convert bulk kinetic energy into thermal energy. The increase, $(3 / 2) k \Delta T$, in thermal energy per particle is of order $m\left(v_{1}-v_{2}\right)^{2}$. Because of the ratio of masses, $\Delta T$ is clearly much greater for the ions than for the electrons. Adiabatic compression increases the electron temperature by a factor of $\left(\rho_{2} / \rho_{1}\right)^{\gamma-1}$, where $\gamma$ is the ratio of specific heats. However, $\rho_{2} / \rho_{1}=(\gamma+1) /(\gamma-1)$ for a strong shock, so for $\gamma=5 / 3$, the electron temperature increases only by a factor of 2.5 . Thus, as long as the preshock temperature, $T_{1}$, is negligible compared to the postshock temperature, $T_{2}$, adiabatic compression will not produce a value of $T_{e 2}$ that is significant compared to $T_{i 2}$. There could also be collisional energy exchange within the shock. As long as the shock is thin, the time spent in the shock will be much less than $t_{e i}\left(T_{e}=\bar{T}\right)$. When $T_{e} \ll \bar{T}, t_{e i}$ is shorter by $\left(T_{e} / \bar{T}\right)^{3 / 2}$, but we will see in $\S 5$ that this has only a small effect at later times. We therefore assume that immediately after the shock, the ions have all of the thermal energy, and that $T_{e 2}=0$.

In a thin shock, it is possible that the ion species will not have time to relax to Maxwellian velocity distributions or to equilibrium with each other through collisions. However, since the contribution per ion to $t_{e i}^{-1}$ is the same for $p$ and $\mathrm{He}^{++}, d T_{e} / d t$ depends only on the mean ion temperature. Furthermore, once the ions reach equilibrium with each other, the rate of change of their temperatures due to collisions with electrons is the same, so they will remain in equilibrium. Thus, for simplicity, we assume a single ion temperature, $T_{i}$.

In the postshock region, the adiabatic equation must be replaced by an energy equation for each species, describing $d T / d t$ due to collisions as well as adiabatic compression. Assuming a single ion temperature reduces the number of energy equations to two: (1) an electron equation,

$$
\frac{d T_{e}}{d t}=\frac{T_{i}-T_{e}}{t_{e i}}+(\gamma-1) \frac{T_{e}}{n} \frac{d n}{d t},
$$

describing the temperature evolution of electrons in a given fluid element, and (2) an equation for the mean temperature of the particles in the same fluid element,

$$
\frac{d \bar{T}}{d t}=(\gamma-1) \frac{\bar{T}}{n} \frac{d n}{d t},
$$

which is obtained by averaging the equations for the individual species.

In principle, there are also separate continuity and momentum equations for each species. However, the Coulomb force prevents charge separation on scales larger than the Debye length, and spherical symmetry precludes electrical currents without charge separation, so the electron and ion velocities are identical, and separating these equations provides no additional information. Furthermore, the total pressure $p$, which appears in the total momentum equation, depends only on the mean temperature $\bar{T}$, which is independent of the extent of electronion equilibration and is governed by the same adiabatic equation as in the case of a single collisional gas. Thus, the fluid equations for the density, pressure, and velocity are unchanged, and are merely supplemented by equation (8), which describes the temperature equilibration. This is important, since the equilibration timescale would otherwise break the self-similarity of the solutions for $\rho$, $p$, and $v$.

Since, in the zero-conduction case, the equations and boundary conditions determining $\rho, p$, and $v$ in all three regions of the self-similar model are unaffected by the replacement of a single collisional gas with a fully ionized plasma, we obtain the exact same solution for these variables. Thus, we can regard Bertschinger's solution as a background in which we can solve for $T_{e}$ according to equation (8).

The self-similar solution has a single length scale, which we take to be the turnaround radius, $r_{\mathrm{ta}}(t)$. In the case of a top-hat perturbation, $r_{\mathrm{ta}} \propto t^{8 / 9}$. We adopt the dimensionless functions from Bertschinger (1985) and write $\rho, p$, and $v$ as functions of $\lambda \equiv r / r_{\mathrm{ta}}$ :

$$
\begin{aligned}
& \rho(r, t)=\rho_{c} \Omega_{b} D(\lambda), \\
& p(r, t)=\rho_{c} \Omega_{b}\left(\frac{r_{\mathrm{ta}}}{t}\right)^{2} P(\lambda), \\
& v(r, t)=\frac{r_{\mathrm{ta}}}{t} V(\lambda) .
\end{aligned}
$$

The scaling in terms of the critical density $\rho_{c}=\left(6 \pi G t^{2}\right)^{-1}$ follows from dimensional analysis, since there is no other density scale far outside the initial top-hat perturbation. The factors of $\Omega_{b}$ are convenient in the $\Omega_{b} \ll 1$ case. When we consider the collisionless dark matter generating the potential well, we will also need the total mass of the system

$$
m_{\mathrm{tot}}(r, t)=\frac{4 \pi}{3} \rho_{c} r_{\mathrm{ta}}^{3} M(\lambda)
$$




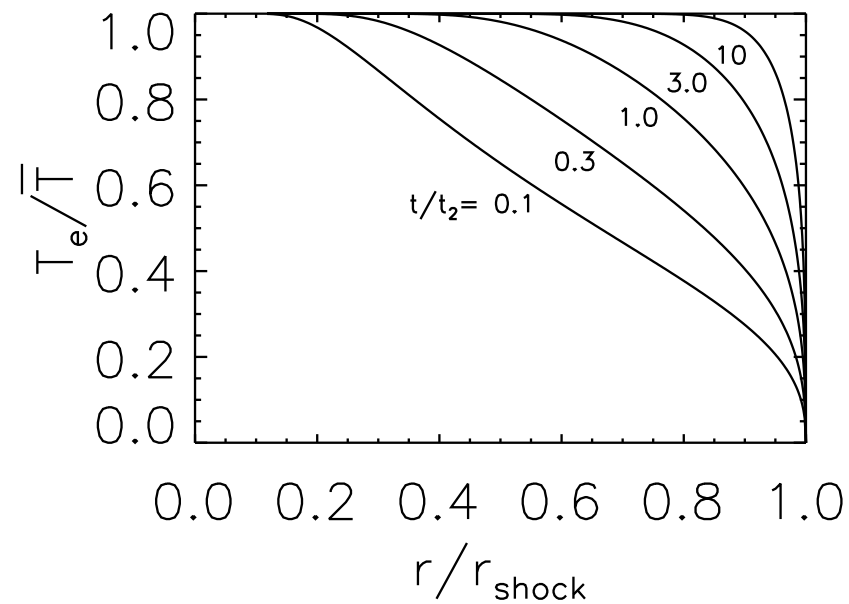

FIG. 1.-Scaled electron temperature $T_{e} / \bar{T}$ as a function of scaled radius $r / r_{\text {shock }}$ for various values of the ratio between the cosmic time $t=t_{0} /$ $(1+z)$ and the electron-ion equilibration time at the shock, $t_{2}$.

\subsection{Electron-Ion Equilibration}

It is useful to introduce scaled temperatures, $\widetilde{T}_{e} \equiv T_{e} / \bar{T}$ and $\widetilde{T}_{i} \equiv T_{i} / \bar{T}$. We then find

$$
\frac{d \tilde{T}_{e}}{d t}=\frac{1}{\bar{T}} \frac{d T_{e}}{d t}-\frac{\tilde{T}_{e}}{\bar{T}^{2}} \frac{d \bar{T}}{d t}=\frac{\tilde{T}_{i}-\tilde{T}_{e}}{t_{e i}},
$$

where the adiabatic compression terms cancel. Note that $t_{e i}$ still depends on $T_{e}=\bar{T} \widetilde{T}_{e}$. However, under the postshock adiabatic conditions, $\bar{T} \propto n_{i}^{\gamma-1} \propto n_{i}^{2 / 3}$, so by coincidence the factor $\bar{T}^{3 / 2} / n_{i}$ is actually a constant for a given fluid element. There is still some residual dependence in the Coulomb term, $\Lambda$, but since $t_{e i}$ depends only logarithmically on $\Lambda$, this dependence can be neglected. We consider any fluid element just after the time, $t_{s}$, when it passed through the shock. It is convenient to define $t_{2 s}$ to be the value that $t_{e i}$ would have had just after the shock if $\widetilde{T}_{e}$ were unity, i.e.,

$$
\begin{aligned}
t_{2 s} & \equiv t_{e i}\left(t_{s}\right) \widetilde{T}_{e}^{-3 / 2}\left(t_{s}\right) \\
& =\frac{7.97 \times 10^{9} \mathrm{yr}}{\ln \Lambda_{2 s}} \frac{\left[\bar{T}_{2}\left(t_{s}\right) / 10^{8} \mathrm{~K}\right]^{3 / 2}}{\left[n_{i 2}\left(t_{s}\right) / 10^{-3} \mathrm{~cm}^{-3}\right]},
\end{aligned}
$$

where $\Lambda_{2 s}$ is, similarly, the value $\Lambda$ would have had just inside the shock radius, if $\tilde{T}_{e}$ were unity. Then, neglecting the slight variation in $\ln \Lambda$,

$$
t_{e i}=t_{2 s} \tilde{T}_{e}^{3 / 2}
$$

and

$$
\frac{d \widetilde{T}_{e}}{d t}=\frac{\widetilde{T}_{i}-\widetilde{T}_{e}}{t_{2 s} \widetilde{T}_{e}^{3 / 2}}
$$

Finally, by definition,

$$
\bar{T} \equiv \frac{n_{e} T_{e}+n_{i} T_{i}}{n_{e}+n_{i}}
$$

and by eliminating $\widetilde{T}_{i}$ from equation (15), we find

$$
\frac{d \widetilde{T}_{e}}{d t}=\frac{1}{t_{2 s}}\left(\frac{n_{i}+n_{e}}{n_{i}}\right)\left(1-\widetilde{T}_{e}\right) \tilde{T}_{e}^{-3 / 2} .
$$

For $\left(1-\tilde{T}_{e}\right) \ll 1$, this simply represents exponential decay of the fractional temperature difference, $\widetilde{T}_{i}-\widetilde{T}_{e}$, with an $e$-folding time of $t_{2 s} n_{i} /\left(n_{i}+n_{e}\right) \approx t_{2 s} / 2$. In fact, equation (17) can be integrated analytically for arbitrary $\widetilde{T}_{e}$ to find $t\left(\widetilde{T}_{e}\right)$. If we neglect the preshock electron temperature as well as energy exchange within the shock and assume $\widetilde{T}_{e 2}=0$ at the time $t_{s}$ when a particular fluid element has just passed through the shock, then

$$
\begin{aligned}
\Delta t \equiv & t-t_{s}=t_{2 s}\left(\frac{n_{i}}{n_{i}+n_{e}}\right) \\
& \times\left[\ln \left(\frac{1+\sqrt{\widetilde{T}_{e}}}{1-\sqrt{\widetilde{T}_{e}}}\right)-2 \sqrt{\widetilde{T}_{e}}\left(1+\frac{\widetilde{T}_{e}}{3}\right)\right] .
\end{aligned}
$$

This solution has precisely the same form found by Shafranov (1957) in the case of a stationary planar shock without postshock adiabatic compression. A nonzero value of $\widetilde{T}_{e 2}$ would be equivalent to adding a correction $\delta t$ to $\Delta t$. Because of the rapid relaxation when $\widetilde{T}_{e} \ll 1$, this correction is generally $\ll t_{2 s}$. Thus at late times, when $\widetilde{T}_{e} \gg \widetilde{T}_{e 2}$, the corresponding correction to $\tilde{T}_{e}(t)$ is small. We will estimate the magnitude of this effect in $\S 5$.

\subsection{Cluster Profile at a Given Time}

We now have an implicit solution for the scaled electron temperature, $\widetilde{T}_{e}$, of a fluid element as a function of the time since that fluid element passed through the shock. For application to X-ray clusters, one is more interested in $\widetilde{T}_{e}$ as a function of radius at a fixed cosmic time, $t$. We consider a fluid element observed at radius $r$ and ask at what time $t_{s}$ it passed through the shock. The shock is characterized by a fixed value, $\lambda_{s}$, of $\lambda=r / r_{\mathrm{ta}}$. For a given fluid element,

$$
\frac{d \lambda}{d \ln t}=t \frac{\dot{r}}{r_{\mathrm{ta}}}-t \frac{r}{r_{\mathrm{ta}}} \frac{\dot{\mathrm{r}}_{\mathrm{ta}}}{r_{\mathrm{ta}}}
$$

Expressing $\dot{r}=v$ in terms of the dimensionless $V=v t / r_{\mathrm{ta}}$ and noting that $t \dot{r}_{\mathrm{ta}} / r_{\mathrm{ta}}=\frac{8}{9}$ for infall onto a top-hat perturbation, we can rewrite equation (19) as

$$
\frac{d \lambda}{d \ln t}=V-\frac{8}{9} \lambda
$$

so that

$$
\frac{t}{t_{s}}=\exp \int_{\lambda}^{\lambda_{s}} \frac{d \lambda}{(8 / 9) \lambda-V} \equiv \epsilon(\lambda) .
$$

The function $\epsilon(\lambda)$ is 1 for $\lambda=\lambda_{s}$ and increases as $\lambda$ decreases. For a fixed $t$, fluid elements at different $r$, and hence different $\lambda$, have different values of $t_{s}$. We are actually interested in

$$
\Delta t=t-t_{s}=t_{s}(\epsilon-1)=t(\epsilon-1) / \epsilon .
$$

The timescale for relaxation is fixed by $t_{2 s}$, which is a constant for each fluid element but varies from one fluid element to the next. Since $t_{2 s} \propto \bar{T}_{2}^{3 / 2} n_{i 2}^{-1}\left(t_{s}\right)$, we see from the scaling of $\rho$ and $p$ at fixed $\lambda$ (eq. [10]) that

$$
\left.t_{2 s} \propto\left(\frac{r_{\mathrm{ta}}}{t}\right)^{3} t^{2}\right|_{t=t_{s}} \propto t_{s}^{5 / 3} \propto \epsilon^{-5 / 3} .
$$

Following equation (18), the scaled temperature, $\tilde{T}_{e}$, is determined by the ratio

$$
\frac{\Delta t}{t_{2 s}}=\frac{t}{t_{2}} \epsilon^{2 / 3}(\epsilon-1) .
$$

where $t_{2}$ is the value of $t_{2 s}$ for the fluid element passing through the shock at time $t$. Combining this with the implicit solution for $\widetilde{T}_{e}(\Delta t)$, and equation (21), we have an implicit solution for $\widetilde{T}_{e}$ as a function of $\lambda<\lambda_{s}$. Given $n_{e} / n_{i}$, $\widetilde{T}_{e}(\lambda)$ is parameterized by the value of the ratio $t / t_{2}$. These 
solutions are shown in Figure 1 for various values of this parameter, using $n_{e} / n_{i}=1.07$ as appropriate for a fully ionized hydrogen-helium plasma with $X=76 \%$. For $t / t_{2} \gg 1$, thermal equilibrium takes place rapidly compared to the dynamical time, and the temperature difference is significant only in a narrow region near $r_{\text {shock}}$, where the fluid elements have passed through the shock very recently. When $t / t_{2} \lesssim 1$, the effect may be significant over a larger fraction of the shock radius. The detailed shape of the curves depends on $\epsilon(\lambda)$, which is determined by the velocity of the fluid elements as they settle toward their equilibrium radii.

\section{MODEL PARAMETERS}

To determine the significance of the lack of electron-ion equilibrium, we need to relate the model parameters to values for typical observed clusters. The self-similar model itself has only one free parameter, $r_{\text {ta }}$, which determines its scale. The mass, density, and temperature all depend on $r_{\text {ta }}$. Unfortunately, X-ray measurements of these quantities tend to come from the inner parts of clusters, where the approximations leading to the self-similar solution break down and the solution has unrealistic divergences. We need to relate an observable quantity in the inner regions of a cluster to a quantity in the outer regions where selfsimilarity holds. One way to do this is to use a simplified model, such as that considered by Eke, Cole, \& Frenk (1996), in which both the X-ray gas and total mass have the form of a singular isothermal sphere in the virialized section of the cluster. In this model, the mass within a mean overdensity of 178 and the temperature of the X-ray gas are related by

$$
m_{178}=2.34 \times 10^{15} h_{50}^{-1} M_{\odot}\left[\left(\frac{T_{\mathbf{X}}}{10^{8} \mathrm{~K}}\right) \frac{1}{(1+z)}\right]^{3 / 2},
$$

where $z$ is the cosmological redshift and we have assumed $X=76 \%$. While this model is obviously an oversimplification, the relationship it predicts between mass and emission-weighted X-ray temperature agrees well with $\mathrm{N}$ body/hydrodynamic simulations (Navarro et al. 1995; Pen 1997). Furthermore, it meets our need to relate an observable quantity to a property of the outer parts of clusters, where self-similarity might hold.

It is easy to show that the overdensity in Bertschinger's collisionless model, given by the dimensionless quantity $M(\lambda) / \lambda^{3}$, equals 178 at $\lambda \approx 0.255$ where $M \approx 2.98$. Using the scaling relation in equation (11), we find

$$
\begin{aligned}
r_{\mathrm{ta}} & =13.2 h_{50}^{-1} \mathrm{Mpc}(1+z)^{-1}\left(\frac{m_{178}}{2 \times 10^{15} h_{50}^{-1} M_{\odot}}\right)^{1 / 3} \\
& =14.0 h_{50}^{-1} \mathrm{Mpc}(1+z)^{-3 / 2}\left(\frac{T_{\mathrm{X}}}{10^{8} \mathrm{~K}}\right)^{1 / 2} .
\end{aligned}
$$

The shock occurs at a fraction $\lambda_{s}=0.3472$ of the turnaround radius, so

$$
\begin{aligned}
r_{s} & =4.60 h_{50}^{-1} \mathrm{Mpc}(1+z)^{-1}\left(\frac{m_{178}}{2 \times 10^{15} h_{50}^{-1} M_{\odot}}\right)^{1 / 3} \\
& =4.84 h_{50}^{-1} \mathrm{Mpc}(1+z)^{-3 / 2}\left(\frac{T_{\mathrm{X}}}{10^{8} \mathrm{~K}}\right)^{1 / 2} .
\end{aligned}
$$

Given $r_{\mathrm{ta}}$ and $t=t_{0}(1+z)^{-3 / 2}$, we can convert the dimensionless quantities $D, P$, and $V$ of the self-similar solution to the physical quantities $\rho, p$, and $v$. In the self-similar model, the temperature $T \propto P / D$, which diverges as $\lambda^{-1 / 4}$ at small $\lambda$. Since we do not expect the self-similar model to be realistic at small radii, we simply cut it off at $\lambda=0.105$, where $T$ becomes greater than $T_{\mathrm{X}}$. For typical cluster conditions, the electrons and ions reach thermal equilibrium outside this radius, and our results depend only on the calibration of $r_{\text {ta }}$, not on the details of the central temperature profile.

In order to calculate the equilibration timescale $t_{2}$, we need the mean temperature $\bar{T}_{2}$, the ion density $n_{i 2}$, and the Coulomb logarithm $\Lambda_{2}$, all evaluated just inside the shock radius. Given the mean molecular weight $\mu m_{p}$ (which we take to be $\mu=0.59$ for $X=76 \%$ ), we can obtain $\bar{T}_{2}$ from $p_{2} / \rho_{2}$. Using Bertschinger's values for $P_{2}$ and $D_{2}$, we find

$$
\bar{T}_{2}=0.44 T_{\mathrm{X}} \text {. }
$$

Similarly, we can find $n_{i 2}$ and $n_{e 2}$ from $\rho_{2}$ :

$$
\left\{\begin{array}{l}
n_{i 2} \\
n_{e 2}
\end{array}\right\}=\left\{\begin{array}{l}
3.74 \\
4.01
\end{array}\right\} \times 10^{-6} \mathrm{~cm}^{-3}\left(\frac{\Omega_{b} h_{50}^{2}}{0.10}\right)(1+z)^{3} .
$$

The Coulomb logarithm, which depends on $\bar{T}$ and $n_{e}$, is then

$$
\ln \Lambda_{2}=39.7+\ln \left(\frac{T_{\mathrm{X}}}{10^{8} \mathrm{~K}}\right)-\frac{1}{2} \ln \left(\frac{\Omega_{b} h_{50}^{2}}{0.10}\right)-\frac{3}{2} \ln (1+z) .
$$

Combining these values, we find

$$
\frac{t}{t_{2}}=0.828 h_{50}^{-1}(1+z)^{3 / 2}\left(\frac{T_{\mathrm{X}}}{10^{8} \mathrm{~K}}\right)^{-3 / 2}\left(\frac{\Omega_{b} h_{50}^{2}}{0.10}\right)\left(\frac{\ln \Lambda_{2}}{39.7}\right)
$$

Measurements of deuterium abundance in quasar absorption systems indicate that $\Omega_{b} h_{50}^{2}=0.096_{-0.020}^{+0.024}$ (Tytler, Fan, $\&$ Burles 1996; Burles \& Tytler 1997). We assume $\Omega_{b} h_{50}^{2}=$ 0.10 .

\section{NUMERICAL CALCULATION}

In order to produce cluster profiles at a fixed time, we need to reproduce the self-similar solutions for $D, P$, and $V$. We integrated the fluid equations for one-dimensional selfsimilar accretion in an external potential, following the same techniques as Bertschinger (1985). Specifically, we integrated Bertschinger's equation (3.4a)-(3.4c), with $M$ replaced with the collisionless $M_{x}$ appropriate for the $\Omega_{b} \ll$ 1 case, using the change of variables given by Bertschinger's equation (3.12) to avoid singularities at the origin. The initial postshock conditions for the integration were given by Bertschinger's equation (3.6), where $V_{1}$ and $D_{1}$, as well as the location, $\lambda_{s}=0.3472$, of the shock, were taken from Bertschinger's Table 10 . For simplicity we calculated the collisionless mass distribution $M_{x}$ by quadratic interpolation of Bertschinger's Table 4, using the scaling $M \propto \lambda^{3 / 4}$ for $\lambda<0.02$.

The equations were numerically integrated inward from the shock using a fifth-order Runge-Kutta routine. Values of $D, P$, and $V$ were calculated on a grid with $\Delta \lambda=\lambda / 500$. The shock location was determined by the condition that the velocity $V$ should be zero at the origin. Because we use values for $M_{x}$ interpolated from a table, we are effectively using a slightly different mass distribution for the collisionless fluid. Thus $\lambda_{s}=0.3472$ is no longer precisely the right 
value. This causes discrepancies with Bertschinger's $\Omega_{b} \ll 1$ solution that are worst near the origin.

Since we cut off our solutions for the electron and ion temperatures at $\lambda=0.105$ where $T=T_{\mathrm{X}}$, only the region outside that radius concerns us. In this region $P / D$, which determines $\bar{T}$, differs from Bertschinger's results by less than $0.5 \%$. While the fractional errors in $V$ reach $7 \%$, the resulting fractional errors in $\epsilon$ are only $0.5 \%$.

We also used the two local integrals of motion given in Bertschinger's equations (2.27) and (3.10) as checks on the calculation. For $\lambda \geq 0.105$, the largest fractional errors in these integrals were $10^{-4}$.

In Figure 2, we show the electron, ion, and mean temperatures as a function of radius for various values of the emission-weighted X-ray temperature. In making comparisons with observed X-ray clusters, the density could in principle be measured directly, rather than calculated from equation (29).

\section{APPROXIMATIONS}

In $\S 2$, we made a number of approximations that we now proceed to justify given the model parameters estimated in $\S 3$. Several of the approximations result in errors near the shock, where the temperature equilibration is rapid owing to the small value of $\widetilde{T}_{e}$. The effect of these errors at later times is equivalent to a change of definition of $\Delta t$ from $t-t_{s}$ to $t-t_{s}-\delta t$. Because the relaxation rate for $\widetilde{T}_{e} \sim 1$ is much smaller than for $\widetilde{T}_{e} \ll 1$, the error $\delta \widetilde{T}_{e} \approx \delta t\left(d \widetilde{T}_{e} / d t\right)$ at later times is generally quite small. One such approximation was
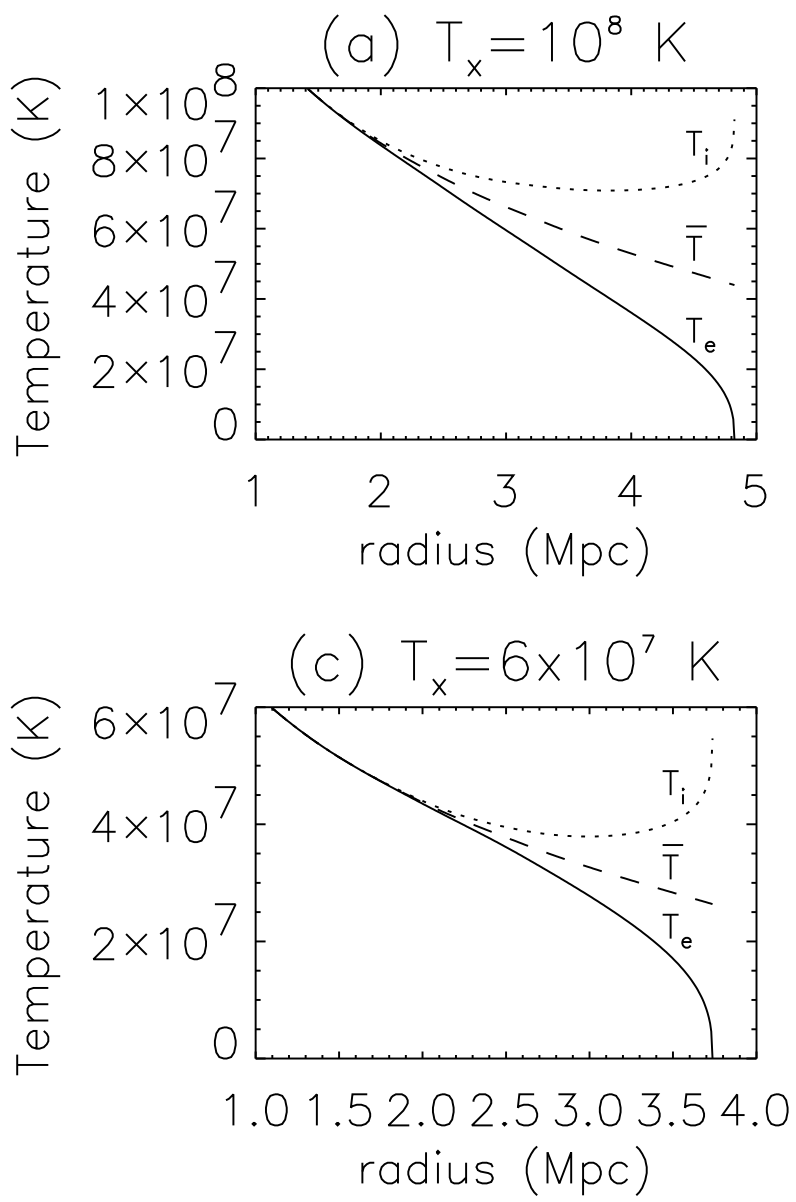

the assumption that $\widetilde{T}_{e}=0$ at $t_{s}$, which neglected the preshock electron temperature as well as energy exchange within the shock. In this case, $\delta t$ is the time it would take for $\widetilde{T}_{e}$ to go from zero to the actual $\widetilde{T}_{e 2}$, which equation (18) shows is

$$
\delta t=t_{2 s}\left(\frac{n_{i}}{n_{i}+n_{e}}\right)\left[\ln \left(\frac{1+\sqrt{\widetilde{T}_{e 2}}}{1-\sqrt{\widetilde{T}_{e 2}}}\right)-2 \sqrt{\widetilde{T}_{e 2}}\left(1+\frac{\widetilde{T}_{e 2}}{3}\right)\right] .
$$

Thus, the fractional error in $\widetilde{T}_{e}$ is

$$
\begin{aligned}
\frac{\delta \widetilde{T}_{e}}{\widetilde{T}_{e}} & =\left[\ln \left(\frac{1+\sqrt{\widetilde{T}_{e 2}}}{1-\sqrt{\widetilde{T}_{e 2}}}\right)-2 \sqrt{\widetilde{T}_{e 2}}\left(1+\frac{\widetilde{T}_{e 2}}{3}\right)\right]\left(1-\widetilde{T}_{e}\right) \tilde{T}_{e}^{-5 / 2} \\
& \approx 0.4\left(1-\widetilde{T}_{e}\right)\left(\frac{\widetilde{T}_{e 2}}{\widetilde{T}_{e}}\right)^{5 / 2}
\end{aligned}
$$

for $\widetilde{T}_{e 2} \lesssim 0.1$. This correction is small except where $\widetilde{T}_{e}$ is of order $\widetilde{T}_{e 2}$.

To determine the significance of this effect, we need to know $\widetilde{T}_{e 2}$. For $\bar{T}_{2}=0.44 T_{\mathrm{X}}$, adiabatic compression alone results in $\tilde{T}_{e 2}=5.7 T_{e 1} / T_{\mathrm{X}}$. Thus, in X-ray clusters with $T_{\mathrm{X}} \gtrsim 10^{7} \mathrm{~K}$, the resulting $\widetilde{T}_{e 2}$ will be small unless the preshock temperature is $\gtrsim 10^{6} \mathrm{~K}$.

The contribution of collisional energy exchange within the shock to $\widetilde{T}_{e 2}$ depends on the detailed shock structure, but we can estimate it. If we repeat the derivation of $\S 2.3$, but ignore adiabatic compression and scale the temperatures in terms of $\bar{T}_{2}$ instead of $\bar{T}$, the factor of $\left(1-\widetilde{T}_{e}\right)$ in
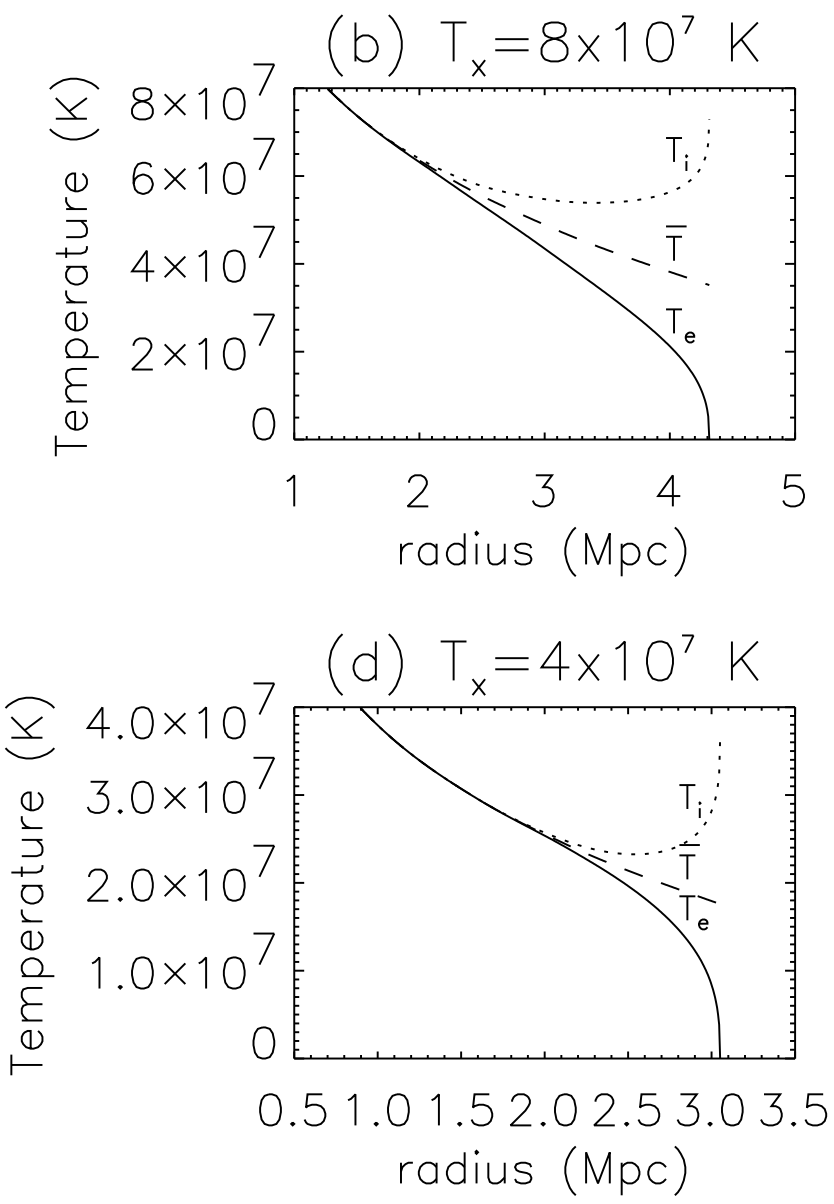

FIG. 2.-Electron temperature (solid line), ion temperature (dotted line), and mean temperature (dashed line) as a function of radius for four clusters with emission-weighted X-ray temperatures $T_{\mathrm{X}}$ of $(a) 10^{8} \mathrm{~K},(b) 8 \times 10^{7} \mathrm{~K},(c) 6 \times 10^{7} \mathrm{~K}$, and $(d) 4 \times 10^{7} \mathrm{~K}$. 
equation (17) is replaced with $\bar{T} / \bar{T}_{2}-\widetilde{T}_{e}$. This reduces $d \widetilde{T}_{e} / d t$ by a factor $\geq \bar{T}_{2} / \bar{T}$. The resulting $\tilde{T}_{e 2}$ is given by equation (32), where now $\delta t$ is the time spent in the shock multiplied by the time average of $\bar{T} / \bar{T}_{2}$. As a rough estimate, suppose this effective time, $\delta t$, equals the postshock proton collision time, $t_{p p 2}$, which results in $\widetilde{T}_{e 2}=0.66$. Figure 3 compares the resulting electron temperature profile with the $\delta t=0$ result for $T_{\mathrm{X}}=10^{8} \mathrm{~K}$. The fractional difference is large at the shock, but quickly becomes smaller than the difference between $T_{e}$ and $\bar{T}$. As a fraction of $\left(1-\widetilde{T}_{e}\right)$, the effect of energy exchange within the shock is less dramatic for lower temperature clusters. We should note that for $\delta t=t_{p p 2}$, the width of the shock is of order $1 \mathrm{Mpc}$ $\times\left(T_{\mathrm{X}} / 10^{8} \mathrm{~K}\right)^{2}$ at $z=0$, which in the hottest clusters is a significant fraction of the shock radius. This will break selfsimilarity and alter the overall density and temperature profiles of the cluster. Figure 3 should only be regarded as an approximate description for the electron temperature in this case. Conversely, as long as a thin shock is a reasonable approximation (e.g., due to embedded magnetic fields), collisional energy exchange within the shock will not significantly alter the results for the electron temperature over most of the region where $T_{e}$ and $T_{i}$ differ.

A second approximation that may fail near the shock is the assumption that $T_{e} / T_{i} \gg m_{e} / m_{p}$. For $\widetilde{T}_{e} \lesssim 10^{-3}$ this breaks down, so the equilibration timescale levels off rather than continuing to decrease as $\widetilde{T}_{e}^{3 / 2}$, and $\widetilde{T}_{e}$ grows only exponentially, rather than as $(\Delta t)^{2 / 5}$. If $\widetilde{T}_{e 2}$ is less than this value, our assumption results in a shift, $\delta t$, opposite in sign to the one above. Given $\widetilde{T}_{e 2}$ estimated before, this is likely to be unimportant. Even for $\widetilde{T}_{e} \lesssim 10^{-3}, t_{e i} \sim 10^{-4} t_{2 s}$, so the time $\delta t$ to go from $T_{e} \sim 10^{3} \underset{\mathrm{K}}{ }$ to $\widetilde{T}_{e}=10^{-3}$ is less than $4 \times 10^{-4} t_{2 s}$. Thus, even in this worst case, $\delta \widetilde{T}_{e}$ remains significant only as long as $\widetilde{T}_{e} \lesssim 10^{-3}$.

A final approximation that causes errors near the shock is our use of $\widetilde{T}_{e}=1 \mathrm{in} \ln \Lambda_{2 s}$. The error introduced depends on the value of $\ln \Lambda_{2 s}$, formally breaking the one-parameter family of solutions shown in Figure 1. However, for the values of $\ln \Lambda_{2 s}$ calculated in $\S 3$ and shown in Figure 2, we can estimate its magnitude. A rigorous upper bound on this effect can be calculated, and for $T_{e 2} \geq 1000 \mathrm{~K}$ it produces $\delta \widetilde{T}_{e} / \widetilde{T}_{e}<5 \%$ for $\widetilde{T}_{e}>0.1$.

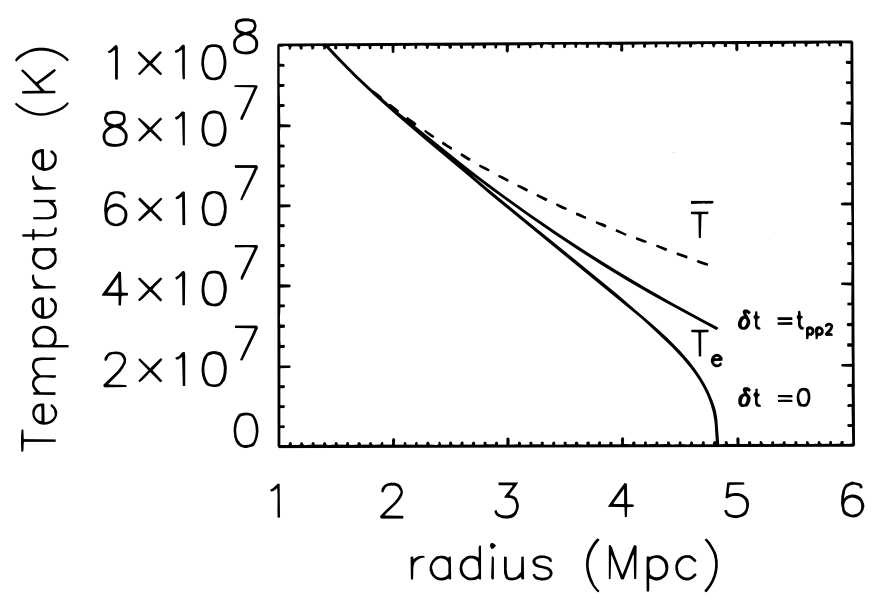

FIG. 3.-Effect of energy exchange within the shock for a cluster with emission-weighted temperature $T_{\mathrm{X}}=10^{8} \mathrm{~K}$. The electron temperature (solid line) assuming an effective time within the shock of one postshock proton-proton collision time $\left(\delta t=t_{p p 2}\right)$ is compared with the case of no exchange $(\delta t=0)$. The mean temperature (dashed line) is also shown. The effect is smaller for lower temperature clusters.
Two other approximations also involve $\ln \Lambda$, but are exact at the shock and become less accurate at smaller radii. These effects also break the one-parameter family of solutions and affect both Figure 1 and Figure 2. First, our scaling for $t_{2 s}$ for a particular fluid element, given in equation (14), ignores the variation of $\Lambda$ with $\bar{T}$ and $n$, as the fluid element is adiabatically compressed. Since $\Lambda \sim$ $\bar{T} / n^{1 / 2} \sim \bar{T}^{1 / 4}$ for adiabatic compression, and since $\bar{T}$ changes only by a factor of 2.2 from the shock to the centrally dominated $T_{\mathrm{X}}$, the fractional effect on $\ln \Lambda$ is less than $0.6 \%$ and the integrated effect is even smaller. Second, the scaling used in equations (23) and (24) to relate $t_{2 s}$ to $t_{2}$ ignores the variation in $\ln \Lambda_{2 s}$ between fluid elements, since it replaces $\ln \Lambda_{2 s}$ with $\ln \Lambda_{2}$. However, for the values of $T_{X}$ used in Figure 2, the maximum fractional error in $\widetilde{T}_{e}$ is $0.1 \%$.

\section{DISCUSSION}

Our model predicts significant differences between the electron and ion temperatures in regions extending up to a third of the way inward from the accretion shock. The effect is greatest in the hottest clusters, where the equilibration time, $t_{e i}$, is longest. Because the equilibration is exponential (except at early times) with a timescale neither much longer nor much shorter than the ages of clusters, it is difficult to predict the extent of nonequilibrium precisely. As more direct information about the physical characteristics of the $\mathrm{X}$-ray gas in the outer parts of clusters becomes available, it will be possible to refine these predictions.

In principle, the ion temperature could be inferred by measuring the width of X-ray lines broadened by the Doppler shift owing to the ion thermal velocity dispersion. The equilibration timescale, $t_{\mathrm{eq}}$, is proportional to $A / Z^{2}$, so the high ionization states that dominate at cluster temperatures should equilibrate with the protons on timescales $\leq t_{p p}$. Thus we can assume a single ion temperature, $T_{i}$. The rms velocity dispersion due to thermal motions along the line of sight is $v_{\mathrm{los}}=\left(k T_{i} / m_{i}\right)^{1 / 2}$, which produces a line with an rms width

$$
\frac{\Delta E_{\mathrm{rms}}}{E}=\frac{v_{\mathrm{los}}}{c}=\frac{1}{c} \sqrt{\frac{k T_{i}}{m_{i}}}=\frac{3.0 \times 10^{-3}}{\sqrt{A}}\left(\frac{T_{i}}{10^{8} \mathrm{~K}}\right)^{1 / 2},
$$

where $A=m_{i} / m_{p}$. In terms of a resolving power, $E / \Delta E$, where $\Delta E$ is the FWHM of a Maxwellian velocity distribution, equation (34) corresponds to

$$
\frac{E}{\Delta E}=1.4 \times 10^{2} \sqrt{A}\left(\frac{10^{8} \mathrm{~K}}{T_{i}}\right)^{1 / 2} .
$$

For the iron lines near $7 \mathrm{keV}$, a resolving power of $E$ / $\Delta E \approx 1000$, or $\Delta E=7 \mathrm{eV}$, would be needed. The microcalorimeters of the XRS instrument on the planned Astro-E satellite have an instrumental $\Delta E \approx 11.5 \mathrm{eV}$. Convolution with the intrinsic width would broaden this to an observed $\Delta E$ of $13.5 \mathrm{eV}$. A high signal-to-noise ratio, as well as an accurate calibration of the instrumental profile, would be necessary to detect this broadening.

The HETG spectrometer on $A X A F$ will reach the necessary resolving power of $500-1000$ at energies $\lesssim 2 \mathrm{keV}$, for observations of point sources. It might therefore be able to measure the widths of absorption lines of $\mathrm{O}$ VIII, Ne $\mathrm{x}$, or $\mathrm{Mg}$ XII in the spectra of quasars located behind the cluster. While the ionization state of these elements is determined by $T_{e}$ rather than $T_{i}$, the populations of these states are still 
likely to be small in the hotter clusters where we expect significant differences between $T_{e}$ and $T_{i}$.

Turbulent line broadening may also be significant in the outer parts of clusters. The ratio of thermal to turbulent broadening is

$$
\frac{v_{\text {los }}}{v_{\text {turb }}}=\left(\frac{c_{S}}{v_{\text {turb }}}\right) \sqrt{\frac{k T_{i} / A m_{p}}{\gamma k T / \mu m_{p}}} \approx \frac{c_{S} / v_{\text {turb }}}{1.7 \sqrt{A}},
$$

where $c_{\mathrm{S}}$ is the sound speed. Widths of several lines of species with $A \lesssim\left(c_{\mathrm{s}} / v_{\text {turb }}\right)^{2}$ would be necessary to distinguish thermal broadening from turbulent broadening.

Fine-structure lines in the ultraviolet might provide another probe of the ion temperature. At the temperatures in question, proton impact excitation can contribute significantly to the population of the upper levels of the Fe XVIII $\lambda 974$ and $\mathrm{Fe}$ XXI $\lambda 1354$ lines (J. Raymond 1997, private communication). Its effect on these lines could therefore be used to measure the ion temperature.

Differences between electron and ion temperatures might be even more significant in clusters formed by mergers than in our simple, spherical accretion model. In a merger, the time since gas was shocked would be smaller, but preheating of the gas would be more important. Three-dimensional hydrodynamic simulations would be essential in exploring the extent of deviations from thermal equilibrium in this case.

We thank Bill Forman, Maxim Markevitch, Ue-Li Pen, and John Raymond for useful discussions. This work was supported in part by a National Science Foundation Graduate Research Fellowship and by NASA GSRP Fellowship NGT-51664 for D. C. F., and by NASA ATP grant NAG 5-3085 and the Harvard Milton fund for A. L. One of the authors (D. C. F.) would like to acknowledge Dragon Systems, Inc., whose DragonDictate for Windows software was essential to the preparation of this paper.

After the completion of this work, we became aware of a preprint by Chièze, Alimi, \& Teyssier (1997) on the same problem. Their results, using a three-dimensional hydrodynamic code to simulate the formation of an X-ray cluster, are consistent with the general conclusions drawn from our semianalytical approach.
Bertschinger, E. 1985, ApJS, 58, 39

Burles, S., \& Tytler, D. 1997, Science, submitted

Chièze, J.-P., Alimi, J.-M., \& Teyssier, R. 1997, preprint

Eke, V. R., Cole, S., \& Frenk, C. S. 1996, MNRAS, 282, 263

Evrard, A. E., Metzler, C. A., \& Navarro, J. F. 1996, ApJ, 469, 494

Itoh, H. 1978, PASJ, 30, 489

Jones, C., \& Forman, W. 1992, in Clusters and Superclusters of Galaxies, ed. A. C. Fabian (Dordrecht: Kluwer), 49

Kim, K.-T., Kronberg, P. P., Dewdney, P. E., \& Landecker, T. L. 1990, ApJ, 355, 29

Kim, K.-T., Tribble, P. C., \& Kronberg, P. P. 1991, ApJ, 379, 80

Markevitch, M., Mushotzky, R., Inoue, H., Yamashita, K., Furuzawa, A., \& Tawara, Y. 1996, ApJ, 456, 437

\section{REFERENCES}

Navarro, J. F., Frenk, C. S., \& White, S. D. M. 1995, MNRAS, 275, 720

Pen, U. 1997, preprint

Rephaeli, Y. 1988, Comments Astrophys., 12, 265

Rosenbluth, M. N. 1965, in Plasma Physics (Vienna: International Atomic Energy Agency), 485

Shafranov, V. D. 1957, Soviet Phys.-JETP Lett., 5, 1183

Spitzer, L. 1962, Physics of Fully Ionized Gases (New York: Wiley)

Tytler, D., Fan, X.-M., \& Burles, S. 1996, Nature, 381, 207

Zeldovich, Y. B., \& Raizer, Y. P. 1967, Physics of Shock Waves and HighTemperature Hydrodynamic Phenomena (New York: Academic) 